\title{
Machine Learning for Automated Analysis of Asbestos Fibres
}

Matt Hiscock ${ }^{1}$, Cecilia Pisano ${ }^{1}$ and Christian Lang ${ }^{2}$

${ }^{1}$ Oxford Instruments NanoAnalysis, United States, ${ }^{2}$ Oxford Instruments Nanoanalysis, High Wycombe, England, United Kingdom

Automated feature analysis is an SEM based process that aims to automatically characterise samples containing potentially large numbers of features (particles/fibres/inclusions) - measuring them in terms of their composition and morphology and classifying the groups of detected particles. The process aims to replicate the tasks that are normally carried out by a human operator during manual analysis. A key task at the beginning of the automated feature analysis process is the detection of features - the task of deciding what should be measured for composition and acquiring morphological data from that feature. The human eye and brain together form a very powerful image capture and processing system which is easily able to distinguish between features - even in relatively complex scenarios. Duplicating this capability for automatic sample detection is a task which in some cases is easily achievable and in others is very difficult. Backscattered electron (BSE) images are typically used for the particle detection as their contrast mechanism helps highlight compositional differences between grains. When samples are flat and polished, it is generally straightforward to set up grey level thresholds to detect features - as can be seen in Figure 1. However, in other cases, particularly where there is significant topography in the sample, it can be a struggle to reliably and consistently detect fibres - even with BSE imaging. An example of this is often seen when there is a need to analyse asbestos. The fibrous nature of this material means that individual fibres often group together to form clumps. As the fibres are often of the same composition, there is little contrast between them in BSE images. Furthermore, as the fibres are clumped, topographical indications of where one fibre begins and another one ends are often sporadic. In addition, we frequently see fibres of similar composition intersecting with or crossing one another. All of these effects make it very difficult to individually threshold asbestos fibres based on their grey level - in many cases multiple fibres are detected as single entities - which can then cause inaccuracies when there is a need to report results such as the number of fibres found in a particular sample. To overcome this problem, we propose an image segmentation approach which detects fibres using a combination of machine learning techniques and traditional computer vision algorithms. With respect to machine learning techniques, we applied generative adversarial networks (GANs) to create a "Morphology Selector" which cleans the initial images of unwanted morphologies (non-elongated particles) and an "Intersections Finder" to identify regions where fibres overlap, with the purpose of splitting them into separate contours properly reconnected in the last step of the process. Accurate results were obtained by training a Pix2Pix GAN to identify particle morphologies, and a CycleGAN to detect overlapping regions. Training datasets of synthetic images were used, containing unwanted morphologies (created using data augmentation tools), and elongated particles (Bezier curves). By leveraging the image translation capability of Pix2Pix and CycleGAN, the Morphology Selector was trained to translate images containing mixed morphologies to corresponding images containing elongated shapes only, whereas the Intersections Finder was trained to translate images containing partially overlapping curves to corresponding images were overlapping regions are highlighted. Finally, a complex contour analysis combined with orientation calculations completes the process of fibre detection by properly reconnecting contours (Reconnection Algorithm). The Morphology Selector, Intersections Finder and Reconnection Algorithm have been tested on real images producing 
good segmentation results which have proved to outperform existing standard approaches in SEM based analysis. An example is shown in Figure 2. We will demonstrate the above with reference to both the simulated and real sample images used throughout the development process and discuss the implications for analysts working in this field along with other potential problems that may be addressed by the approach.

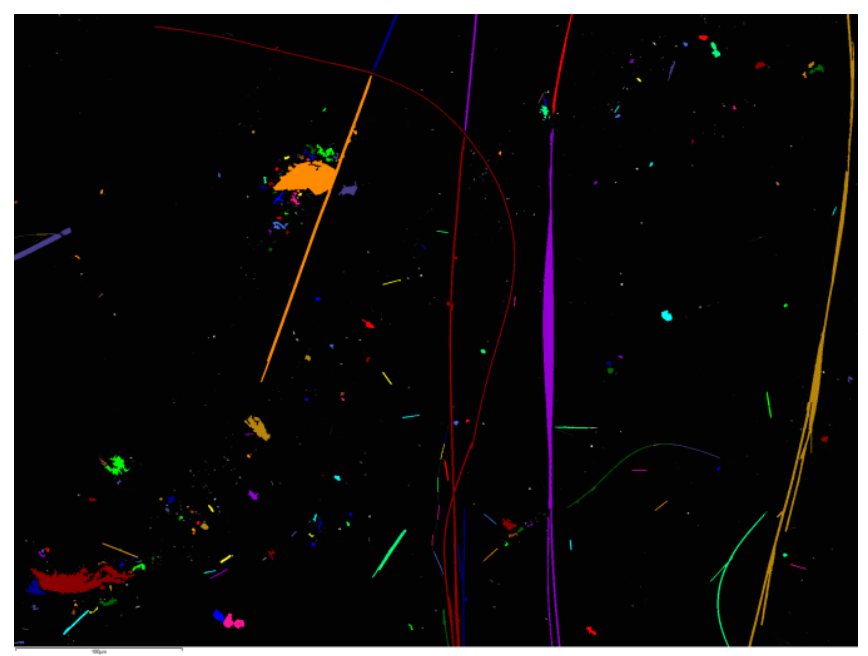

Figure 1. Example of thresholding of asbestos fibres from BSE images. Different colours are assigned to each fibre so that those detected as one can be seen. The image shows several examples where multiple fibres are detected as a single fibre and where single fibres are broken into multiple fibres by other fibres crossing them.

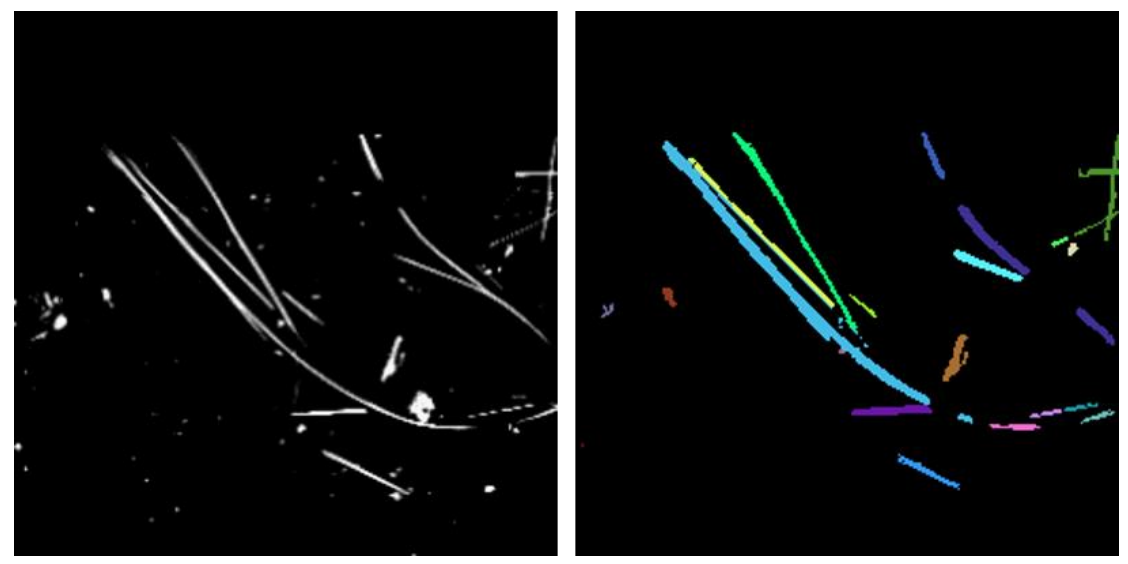

Figure 2. Field of view containing asbestos fibres pre and post processing by machine learning approach. Fibres which could otherwise be detected with the problems seen above are far more clearly resolved. 